\title{
Exploring the effects of galacto-oligosaccharides on the gut microbiota of healthy adults receiving amoxicillin treatment
}

\author{
S. E. Ladirat ${ }^{1}$, M. H. C. Schoterman ${ }^{2}$, H. Rahaoui ${ }^{3}$, M. Mars ${ }^{4}$, F. H. J. Schuren ${ }^{3}$, H. Gruppen ${ }^{1}$, \\ A. Nauta $^{2}$ and H. A. Schols ${ }^{1 *}$ \\ ${ }^{1}$ Laboratory of Food Chemistry, Wageningen University, Bornse Weilanden 9, 6708 WG Wageningen, The Netherlands \\ ${ }^{2}$ FrieslandCampina, Stationsplein 4, 3818 LE Amersfoort, The Netherlands \\ ${ }^{3}$ TNO Microbiology \& Systems Biology, Utrechtseweg 48, 3704 HE Zeist, The Netherlands \\ ${ }^{4}$ Division of Human Nutrition, Wageningen University, PO Box 8129, 6700 EV Wageningen, The Netherlands
}

(Submitted 21 November 2013 - Final revision received 2 April 2014 - Accepted 22 April 2014-First published online 13 June 2014)

\begin{abstract}
In the present double-blind, randomised, parallel intervention study, the effects of the intake of galacto-oligosaccharides (GOS) on the gut microbiota of twelve healthy adult subjects (aged $18-45$ years with a normal BMI $\left(18-25 \mathrm{~kg} / \mathrm{m}^{2}\right)$ ) receiving amoxicillin (AMX) treatment were determined. All the subjects were treated with AMX $(375 \mathrm{mg}$; three times per d) for $5 \mathrm{~d}$ and given either GOS ( $n$ 6) or placebo (maltodextrin, $n$ 6) ( $2.5 \mathrm{~g}$; three times per d) during and $7 \mathrm{~d}$ after AMX treatment. Faecal samples were collected twice before starting the treatment and on days 2, 5, 8, 12, 19 and 26. Due to AMX treatment, a decrease in the abundance of Bifidobacterium spp., an overgrowth of Enterobacteriaceae, and a disruption of the metabolic activity of the microbiota (increase in succinate, monosaccharide and oligosaccharide levels in the faecal samples) were observed in both groups $(P<0.05)$. Positive effects of GOS intake were observed on the levels of bifidobacteria, although not found to be significant. Data revealed that the levels of bifidobacteria were higher upon GOS intake than upon placebo intake, especially after AMX treatment. The activity of bifidobacteria and subsequent cross-feeding activity of the microbiota upon GOS intake compared with those upon placebo intake were reflected by the significant increase in butyrate levels $(P<0.05)$ in the faecal samples after AMX treatment. Despite the small number of subjects, our findings confirm previous results obtained in vitro, namely that GOS intake supports the recovery of the beneficial bifidobacteria and, indirectly, the production of butyrate after AMX treatment.
\end{abstract}

Key words: Galacto-oligosaccharides: Antibiotics: Intestinal microbiota: Human intervention studies

In Europe, the median consumption of antibiotics was $18 \cdot 3$ defined daily doses per 1000 inhabitants per d in $2010^{(1)}$. The main side effect of these medicines is antibiotic-associated diarrhoea, which occurs in $5-10 \%$ of outpatient cases and $10-35 \%$ of inpatient cases. Among these, the antibiotic amoxicillin (AMX) is responsible for the highest incidence of antibiotic-associated diarrhoea ${ }^{(2)}$. Prebiotics, defined as 'selectively fermented ingredients that allow specific changes, both in the composition and/or activity of the gastrointestinal microflora that confer benefits upon host well-being and health'(3), have been suggested to prevent this common side effect of antibiotics. However, literature data concerning the prevention of antibiotic-associated diarrhoea are not consistent $^{(4,5)}$, probably due to the variability in parameters among the studies. Parameters such as prebiotics, antibiotics, dosages and subject age are indeed known to have a high impact on the composition of the microbiota ${ }^{(6)}$.

Recently, an in vitro study using a fermentation screening platform has allowed the straightforward comparison of the impact of four often used antibiotics on the microbiota of healthy adults with and without the intake of galacto-oligosaccharides (GOS), a known prebiotic ${ }^{(7)}$. This study revealed that the recovery of bifidobacteria upon GOS intake is antibioticand dose-dependent. For instance, GOS did not affect the recovery of bifidobacteria after clindamycin treatment, whereas GOS positively affected the recovery of bifidobacteria after AMX treatment. Further understanding of the impact of GOS on the gut microbiota in vivo is essential as the microbiota is considered a key factor in human health ${ }^{(8)}$. Besides their action on the composition on the microbiota,

Abbreviations: AMX, amoxicillin; GOS, galacto-oligosaccharides; MD, maltodextrin; OA, organic acids; SAM, significance analysis of microarrays. 
antibiotics are known to negatively affect the metabolic activity of the microbiota as well ${ }^{(9)}$. The accumulation of monosaccharides and high levels of lactate and succinate have been observed during in vitro fermentation using AMX-disrupted microbiota supplemented with $\mathrm{GOS}^{(7)}$. However, the metabolic activity of antibiotic-treated microbiota upon GOS intake in vivo has never been addressed. Most in vivo studies have investigated the effect of fructooligosaccharides and focused on the frequency of diarrhoea and the prevention of Clostridium difficile infection.

In the present intervention study, we determined the effects of GOS intake on the microbiota of healthy adult subjects during and after treatment with AMX to investigate whether trends observed in a previous in vitro study ${ }^{(7)}$ represent the situation in healthy humans as well. The impact of GOS on the composition of the microbiota was assessed by quantifying changes in the number of total bacteria and bifidobacteria and by interpreting the bacterial fingerprints obtained with a phylogenetic microarray. The impact of GOS on the metabolic activity of the microbiota was assessed by measuring the levels of SCFA, monosaccharides and oligosaccharides in the faecal samples.

\section{Subjects and methods}

\section{Subjects}

A total of twelve subjects were recruited in Wageningen (The Netherlands) and surrounding areas. The subjects were aged $18-40$ years, had a normal BMI $\left(18 \cdot 5-25 \cdot 0 \mathrm{~kg} / \mathrm{m}^{2}\right)$ and consumed a Western diet.

Due to the exploratory nature of the study, no statistical examination of the number of participants was done. Based on other studies that have investigated the activity of the microbiota $^{(10-13)}$, the selected number of twelve volunteers was considered sufficient to validate whether trends observed in previous in vitro studies could be detected in healthy humans as well. This number allows an initial evaluation of the response to the treatment considering individual variation in the microbiota.

Subjects were excluded if they smoked, used drugs, had gastrointestinal diseases or had family members who had the diseases, travelled to Asian, African or Latin American countries in the last 6 months, had hypersensitivity or allergies to the products used in the study (amoxicillin and lactose), had history of allergies, or had hepatic disease or renal failure. Subjects were also excluded if they used medications other than $N$-acetyl- $p$-aminophenol (paracetamol) or acetylsalicylic acid (aspirin), used antibiotics in the last 3 months, had undergone more than three antibiotic treatments in the last 2 years, or consumed prebiotics or probiotics (a list of products was provided) in the last month before the study. Female subjects were also excluded if they used contraceptive pills, were pregnant (including planning to be), gave birth in the last 6 months or were lactating. The study was conducted according to the guidelines laid down in the Declaration of Helsinki, and all procedures involving human subjects were approved by the Medical Ethics Committee of Wageningen University (registration no. NL 42438.018.12). Written informed consent was obtained from all the subjects. The study was registered in the US National Institutes of Health clinical trial database (ClinicalTrials.gov no. NCT01848535).

\section{Supplements}

The antibiotic Amoxicilline disper tablet 375 (Sandoz BV) was provided by a local pharmacy. AMX was investigated in the present study because of its risk of causing diarrhoea and its frequent use ${ }^{(14)}$. A low dose of AMX $(375 \mathrm{mg}$; three times per d) and the shortest duration ( $5 \mathrm{~d}$ ) of treatment were chosen to limit the risks of side effects in the volunteers. The prebiotic Vivinal ${ }^{\circledR}$ GOS (FrieslandCampina Domo) was specified by the supplier to have a DM of $75 \%(w / w)$, of which $59 \%(\mathrm{w} / \mathrm{w})$ was present as GOS, $21 \%(\mathrm{w} / \mathrm{w})$ as lactose, $19 \%$ $(\mathrm{w} / \mathrm{w})$ as glucose and $1 \%(\mathrm{w} / \mathrm{w})$ as galactose. The degree of polymerisation of the oligosaccharides in Vivinal ${ }^{\circledR}$ GOS ranged from 2 to 8 . The dose of Vivinal ${ }^{\circledR}$ GOS (2.5 g; three times per d) was chosen to be sufficient to have a bifidogenic effect and to be low enough to limit gastrointestinal discomfort, such as flatulence ${ }^{(15)}$. The placebo, maltodextrin (MD) SPG 30 (powder; AVEBE U.A.), was specified by the supplier to contain $6 \%(\mathrm{w} / \mathrm{w})$ glucose, $15 \%(\mathrm{w} / \mathrm{w})$ maltose, $18 \%(\mathrm{w} / \mathrm{w})$ maltotriose, $8 \%(\mathrm{w} / \mathrm{w})$ maltotetraose, $12 \%(\mathrm{w} / \mathrm{w})$ maltopentaose, $17 \%(\mathrm{w} / \mathrm{w})$ maltohexaose and $24 \%(\mathrm{w} / \mathrm{w})$ of higher oligosaccharides. The degree of polymerisation of the placebo was similar to that of GOS. The prepared MD syrup had a DM of $75 \%(\mathrm{w} / \mathrm{v})$ and a density of $1 \cdot 2$. The differences in the sweetness and viscosity of the prebiotic and placebo syrups were masked upon solubilisation in $150 \mathrm{ml}$ of orange juice.

\section{Experimental design}

The present study was a double-blind, randomised, parallel intervention study comprising $12 \mathrm{~d}$ of intervention and $14 \mathrm{~d}$ of follow-up (Fig. 1). The twelve subjects were randomly divided into two groups: the first group received AMX and GOS treatment ( $n$ 6), while the second group received AMX and placebo treatment ( $n$ 6). AMX $(375 \mathrm{mg}$; three times per d) was given for $5 \mathrm{~d}$. GOS or placebo $(2.5 \mathrm{~g}$; three times per d) solubilised in $150 \mathrm{ml}$ of orange juice was given along with AMX for $5 \mathrm{~d}$ and was continued to be given for $7 \mathrm{~d}$ after the treatment with AMX was discontinued. The intervention

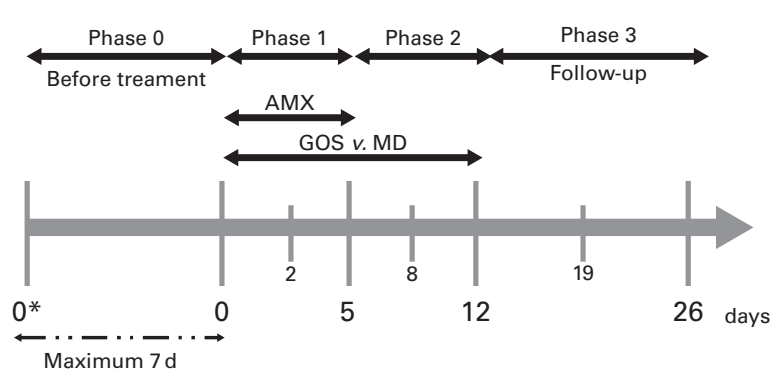

Fig. 1. Scheme of the intervention study. $0^{*}$ refers to samples collected at screening (1-7d before day 0). AMX, amoxicillin; GOS, galacto-oligosaccharides; MD, maltodextrin (placebo). 
products were consumed at breakfast, lunch and dinner. The effects of GOS or placebo intake were monitored up to $14 \mathrm{~d}$ after the intake was discontinued. This follow-up period will also enable to investigate the resilience of the microbiota after the intervention period.

\section{Faecal sample collection and storage}

Faecal samples were collected at eight occasions: at screening (day 0*); day 0; day 2; day 5; day 8; day 12 ; day 19 ; day 26 . The subjects were free to deliver the faecal sample on the indicated day or the day after and were free to deliver the faecal sample at home or at work. After delivering the first faecal sample (day $0^{*}$ ), the subjects had the opportunity to drop out from the study if they found the procedure too invasive. If the subjects wanted to continue participating in the study, they were free to deliver day 0 faecal sample within $7 \mathrm{~d}$ of delivering day $0^{*}$ sample. The subjects started the antibiotic treatment and GOS/placebo intake after delivering day 0 faecal sample. Samples collected on day $0^{*}(1 \times)$ and on day $0(1 \times)$ were used as the baseline samples to estimate the individual variability in the composition and activity of the microbiota.

During each occasion (except on day 0), the subjects dropped the faecal sample in a specimen collection container placed on the toilet seat, collected part of the faecal sample using a spoon and gloves, and placed it in one plastic cup $(120 \mathrm{ml})$. The subjects filled three plastic cups using the same faecal sample up to maximum $60 \mathrm{ml}$. In case of limited availability of faecal material, the faecal sample was equally divided among the three cups. After collecting the faecal samples, the subjects placed the three cups in the coolest environment possible (usually $4^{\circ} \mathrm{C}$ ) and immediately phoned the investigator, who collected the cups within half an hour and stored them at $-80^{\circ} \mathrm{C}$. The first cup was used for DNA isolation, the second cup was used for faecal monosaccharide and oligosaccharide and SCFA measurement, and the last cup was used to carry out a potential failed analysis again. On day 0 , an anaerobic sachet was placed in the specimen collection container, roughly separated from the faecal sample by a plastic layer. The subjects closed the container air-tight, placed it at $4{ }^{\circ} \mathrm{C}$ and immediately phoned the investigator, who collected the container within half an hour. To carry out further fermentation studies, the faecal sample collected on day 0 was processed in an anaerobic chamber. Part of the faecal sample was divided among three cups as described above, and part was suspended in modified standard ileal efflux medium ${ }^{(16)}$ containing $12 \%(\mathrm{v} / \mathrm{v})$ glycerol. The faecal suspension and the cups were stored at $-80^{\circ} \mathrm{C}$.

\section{Stool parameters, gastrointestinal discomfort and adverse events}

The subjects kept a diary to record compliance to the treatment, defecation frequency and stool consistency, gastrointestinal discomfort and adverse events ${ }^{(17,18)}$. Compliance to the treatment was reported by circling 'yes' or 'no' for the intake of antibiotics and/or the orange juice at breakfast (07.00-09.00 hours), lunch (12.00-14.00 hours) and dinner (18.00-21.00 hours). A number was indicated to report defecation frequency. The Bristol scale was provided to the subjects and they used it to score the stool consistency from type 1 (separate hard lumps) to type 7 (watery with no solid pieces). A five-point scale ranging from 1 (not at all) to 5 (very much) was used to score the markers of discomfort (flatulence, bloating, heartburn and nausea). Free space was left to report other discomforts, medicine used, adverse events and other remarks.

An adverse event expected in the study due to the use of amoxicillin was diarrhoea. Diarrhoea is defined by the World Health Organization $^{(19)}$ as the condition of having three or more loose or liquid bowel movements per $\mathrm{d}$. Based on this definition, the frequency and consistency scores were considered serious if the subjects reported $\geq 3$ bowel movements per $\mathrm{d}$ and/ or scored a stool consistency $\geq$ type 6 on the Bristol scale. When a subject reported a serious score, the general practitioner was informed and the practitioner decided whether the subject should be withdrawn from the study or not. If a subject scored a stool consistency of type 6 for $3 \mathrm{~d}$ in a row, the subject was automatically withdrawn from the study and followed by the general practitioner until the symptoms abated. The Medical Ethics Committee was informed about the withdrawal.

\section{DNA isolation}

Faecal sample collected in the first cup was thawed overnight at $4^{\circ} \mathrm{C}$ and homogenised by manual stirring. Total faecal DNA was isolated as described by Crielaard et al. ${ }^{(20)}$ with some minor adjustments: the faecal sample $(100 \mathrm{mg})$ was mixed with $250 \mu \mathrm{l}$ of lysis buffer (Agowa) and $250 \mu \mathrm{l}$ of zirconium beads $(0.1 \mathrm{~mm})$ and $200 \mu \mathrm{l}$ of phenol, before being introduced into a BeadBeater (BioSpec Products) for 2 min. Because of the high levels of impurities in the faeces, phenol extraction was carried out twice. DNA isolation was carried out in duplicate (from the same cup) for the samples of subjects 11 and 12 on days $0,5,12$ and 26 to determine the homogeneity of the sample collected in the first cup.

\section{Microbiota composition analysis}

The Intestinal (I)-Chip, developed at TNO (Zeist, The Netherlands), was used to investigate the composition of the microbiota. This DNA-based microarray enabled the detection of more than 400 bacterial targets from the human large intestinal microbiota. The total faecal DNA isolated was amplified, purified and hybridised as described previously ${ }^{(16)}$. The hybridisation was carried out on a microarray constructed and validated as described previously ${ }^{(20)}$, using intestinal bacterial primers instead of oral primers. ImaGene 5.6 software (BioDiscovery) was used to analyse the results. Genes with a signal intensity greater than 3 $\left(>10^{5}\right.$ bacteria) in more than ten individual samples were used to describe the bacterial fingerprint.

\section{Total bacteria and Bifidobacterium spp. quantification}

The quantification of total bacteria and Bifidobacterium spp. in the faecal samples was done as described previously using quantitative $\mathrm{PCR}^{(7)}$. 


\section{Water-extractable carbohydrate and} organic acid extraction

The extraction of water-extractable carbohydrates and organic acids (OA) was carried out according to the methods of Albrecht et $a l .{ }^{(21)}$ and Jonathan et al. ${ }^{(22)}$, respectively, with minor modifications. The thawed faecal samples were $20 \times$ diluted $(\mathrm{w} / \mathrm{v})$ with Millipore water ( $250 \mathrm{mg}$ in $5 \mathrm{ml}$ of Millipore water). The diluted faecal slurry was centrifuged $\left(3500 \mathrm{~g}, 15 \mathrm{~min}, T=4^{\circ} \mathrm{C}\right)$. An aliquot of the supernatant $(1 \mathrm{ml})$ was boiled $(5 \mathrm{~min})$ to inactivate the enzymes and filtered through a $0.22 \mu \mathrm{m}$ membrane. The faecal extract obtained was used for monosaccharide and oligosaccharide analysis and OA analysis.

\section{Faecal monosaccharide and oligosaccharide quantification}

High-performance anion-exchange chromatography was used to quantify the monosaccharides and oligosaccharides present in the faecal extracts. An ICS5000 HPLC system (Dionex) equipped with a CarboPac PA-1 column ( $2 \mathrm{~mm}$ inner diameter $\times 250 \mathrm{~mm}$; Dionex) in combination with a CarboPac PA guard column ( $2 \mathrm{~mm}$ inner diameter $\times 25 \mathrm{~mm}$ ) and an ISC5000 ED detector (Dionex) in the pulsed amperometric detection mode was used. A flow rate of $0.3 \mathrm{ml} / \mathrm{min}$ was used with the following gradient of $0 \cdot 1 \mathrm{~m}$-sodium hydroxide $(\mathrm{NaOH})$ and $1 \mathrm{~m}$-sodium acetate $(\mathrm{NaOAc})$ in $0 \cdot 1 \mathrm{M}-\mathrm{NaOH}: 0-3 \mathrm{~min}, 20-50 \mathrm{~mm}-\mathrm{NaOH}$; 3-12 min, $\quad 50-75 \mathrm{~mm}-\mathrm{NaOH} ; \quad 12-15 \mathrm{~min}, \quad 100 \mathrm{~mm}-\mathrm{NaOH}$; 15-35 min, 0-200 mm-NaOAc in $0.1 \mathrm{M}-\mathrm{NaOH} ; \quad 35-50 \mathrm{~min}$ washing step with $1 \mathrm{~m}-\mathrm{NaOAc}$ in $0.1 \mathrm{~m}-\mathrm{NaOH}$; $50-53 \mathrm{~min}$, $100 \mathrm{~mm}-\mathrm{NaOH} ; \quad 53-68 \mathrm{~min}$ equilibration with $20 \mathrm{~mm}-\mathrm{NaOH}$. For the quantification of faecal monosaccharides and oligosaccharides, $10 \mu \mathrm{l}$ of a sample were injected onto the column each time. Roughly, the monomers were considered to elute between 0 and 12 min, while the oligosaccharides (including disaccharides) were considered to elute between 15 and $35 \mathrm{~min}$. Lactose was used as a quantification reference. The monosaccharide and oligosaccharide levels are, thereby, expressed in mg saccharides (lactose equivalent) $/ \mathrm{g}$ faeces. Under the conditions mentioned above, lactose $(0.01 \mathrm{mg} / \mathrm{ml})$ had a peak area of $22 \mathrm{nC} / \mathrm{min}$.

\section{Organic acid analysis}

High-performance liquid chromatography was carried out to quantify SCFA (acetate, propionate, butyrate and valerate) and intermediate OA (lactate and succinate) present in the faecal extracts. A Dionex Ultimate 3000 HPLC system (Dionex) equipped with an ion-exclusion Aminex HPX-87H column $(7.8 \times 300 \mathrm{~mm})$ combined with a guard column (Bio-Rad) and an RI-101 refractive index detector (Shodex) was used. The mobile phase was $5 \mathrm{~mm}-\mathrm{H}_{2} \mathrm{SO}_{4}$ and the flow rate was $0.6 \mathrm{ml} / \mathrm{min}$ at $65^{\circ} \mathrm{C}$. The samples $(10 \mu \mathrm{l})$ were injected onto the column. Standards of known concentration $(0 \cdot 25-2 \mathrm{mg} / \mathrm{ml})$ were used for quantification.

\section{Statistical analysis}

Mixed-model ANOVA was used with day and treatment as the fixed factors and subjects as the random factors
(SPSS Statistics 21; IBM). Statistical significance was set at $P<0 \cdot 05$. In case of significant effects, least significant difference was used for post hoc analyses.

The data matrix of bacterial fingerprints obtained with the I-Chip was analysed with significance analysis of microarrays (SAM) to identify markers significantly different between the predefined groups and with a hierarchical clustering based on Euclidean distances (TM4 software) ${ }^{(23)}$. SAM is a statistical technique for finding significant genes in a set of microarray experiments. The input to SAM is gene expression measurements from a set of microarray experiments, as well as a response variable from each experiment. In the present study, the response variables were in the first time the groupings 'GOS group' $v$. 'placebo group' to determine the effect of GOS and in the second time 'phase 0' $v$. 'phase 1' to determine the effect of AMX in the GOS and placebo groups. SAM computes a statistic $d_{i}$ for each gene $i$, measuring the strength of the relationship between gene expression and the response variable. It uses repeated permutations of the data to determine whether the expression of any gene is significantly related to the response. In a two-class unpaired design as that used in the present study, $d$ is analogous to the $t$ statistic in a $t$ test. The cut-off for significance is determined by a tuning parameter delta, chosen by the user based on the false positive rate $(=0)$, which is the proportion of genes likely to have been identified by chance as being significant.

The statistical analysis was carried out before breaking the treatment codes. The values are expressed as means with their standard errors. Due to the small number of subjects, we evaluated not only statistically significant differences, but also individual trends.

\section{Results}

\section{Population characteristics}

The faecal samples of two of the twelve recruited subjects were not analysed because of non-compliance to the treatment assigned: one subject forgot to drink the juices three times and take the antibiotic tablet twice and the other subject forgot to take the antibiotic tablet once. The study population, therefore, consisted of five men and five women who were aged 26 (SEM 4) years and had a BMI of 22.7 (SEM 1.9) kg/m².

\section{Effect of galacto-oligosaccharides on stool parameters and gastrointestinal discomforts}

During phase 0 (before the treatment), the mean defecation frequency (1.6 (SEM 0.2)) and stool consistency on the Bristol scale (type 3.4 (SEM 0.2)) of both groups were similar. Overall, the defecation frequency and stool consistency were constant over $26 \mathrm{~d}$ and hence did not differ statistically. On an individual level, the frequency and consistency scores tended to increase (up to three bowel movements per $\mathrm{d}$ and up to a consistency of type 5 on the Bristol scale) during phase 1 (AMX + GOS/MD) in three subjects (two from the placebo group and one from the GOS group), before returning to the initial values during phase 2 (GOS/MD). The frequency and consistency scores changed noticeably $(\geq 3$ bowel movements per $d$ and/or 
consistency $\geq$ type 6 on the Bristol scale) in two subjects from the GOS group during AMX treatment (phase 1): one subject reported one loose bowel movement of type 6 on days 2 and 3 and the other subject reported three loose bowel movements of type 6 and type 7 on days 4 and 5. Based on the definition of the World Health Organization, the latter subject had diarrhoea and was withdrawn from the study after day 5. The dataset of this subject (days 0-5) was included in the study as it might provide insights into why the subject suffered from diarrhoea.

The effects on the markers of gastrointestinal discomforts, such as flatulence and bloating, were mild (maximum scores of 2-3 on the five-point scale). No differences were observed between the GOS and placebo groups.

\section{Effect of galacto-oligosaccharides on the composition of the microbiota}

The effects of GOS on the composition of AMX-disrupted microbiota were studied by quantifying the levels of total bacteria and Bifidobacterium spp. and by interpreting the bacterial fingerprints obtained with the I-Chip.

Quantification of total bacteria and Bifidobacterium spp. During phase 0 (before the treatment; days $0^{*}$ and 0 ), the numbers of total bacteria and Bifidobacterium spp. were similar in both groups, about $10^{103}$ copies/g faeces and $10^{95}$ copies/g faeces, respectively. Individual variation in the numbers of total bacteria and Bifidobacterium spp. before the treatment is summarised in Table 1 . In the placebo group, one subject had a much lower proportion of Bifidobacterium spp. $(<1 \%)$ when compared with the other subjects (approximately 9\%).

In Fig. 2(a) and (b), the variation in the normalised levels of total bacteria and Bifidobacterium spp. during the study is shown, respectively. The number of total bacteria was not found to differ significantly either per $\mathrm{d}$ or between the treatments. The number of Bifidobacterium spp. was found to differ per $\mathrm{d}(P<0 \cdot 001)$. However, no significant interaction was observed between treatment and time $(P=0.09)$. During phase 1 (AMX + GOS/MD; days 2 and 5), the number of Bifidobacterium spp. decreased in both groups over time due to AMX treatment $(P<0 \cdot 01)$. Furthermore, data revealed that the decrease in the number of Bifidobacterium spp. was lower in the GOS group than in the placebo group on day 5. During phase 2 (GOS/MD; days 8 and 12), the number of Bifidobacterium spp. was higher in the GOS group than in the placebo group on both days 8 and $12\left(+0 \cdot 8 \log _{10}\right.$ and $+1 \log _{10}$, respectively). In the GOS group, the number of Bifidobacterium spp. was higher on day 12 than on day $0\left(+0.7 \log _{10}\right)$. During phase
3 (follow-up period; days 19 and 26), the number of Bifidobacterium spp. was similar to the initial value in both the GOS and placebo groups.

Bacterial fingerprints of the overall microbiota. The bacterial fingerprint of each subject during phase 1 (before the treatment; days $0^{*}$ and 0 ) is shown in Fig. 3. Similar bacterial fingerprints were obtained for the second baseline samples for each subject, indicating good stability of the microbiota within $7 \mathrm{~d}$. The main bacterial groups present in the microbial population of each subject were Bacteroides, Bifidobacterium, Escherichia, Lachnospiraceae, Lactobacillus and Peptostreptococcaceae. On an individual level, differences could be detected in both groups at the species level, especially for Bacteroides, Bifidobacterium and Akkermansia. Overall, the GOS group had an initial microbial population that was richer in Bifidobacterium spp., Bacteroides uniformis and Alistipes putredinis compared with the placebo group (Fig. 4(a)). The difference in the levels of bifidobacteria was solely due to the low levels of bifidobacteria in subject 7 . When the dataset of this subject was excluded from the SAM, no difference in the levels of bifidobacteria could be detected between the two groups.

During the treatment, the impact on the overall microbiota was highly individual dependent. Some trends could be detected by the SAM between the GOS and placebo groups as well as between the phases of the treatment. During phase 1 (AMX + GOS/MD; days 2 and 5), the relative abundance of certain Lachnospiraceae was lower on days 2 and 5 than on day 0 in both groups (Fig. 5(a) and (b)). The relative abundance of some Enterobacteriaceae also changed: the abundance increased significantly on days 2 and 5 than on day 0 in the GOS group (Fig. 4(a)) and in three of the five subjects in the placebo group, despite not being concluded to be significant (see online supplementary Fig. S2). The changes in the relative abundance of certain Enterobacteriaceae and Lachnospiraceae observed in both groups were probably due to the action of AMX. The effects of GOS intake were detected on the number of Bifidobacterium spp., which was significantly higher in the GOS group than in the placebo group (Fig. 4(b)). During phase 2 (GOS/MD; days 8 and 12), the relative abundance of Bifidobacterium spp., B. longum and B. thermophilum was significantly higher in the GOS group than in the placebo group (Fig. 4(c)). During phase 3 (follow-up period; days 19 and 26), while the composition of the microbiota in most of the subjects returned to its initial state, higher levels of Bifidobacterium spp., B. longum and Bacteroides vulgatus were detected in the GOS group than in

Table 1. Number of total bacteria and Bifidobacterium spp. before the treatment (day $0^{*}$ and day 0 ) (Mean values of $\log _{10}$ copies/g faeces with their standard errors)

\begin{tabular}{|c|c|c|c|c|c|c|c|c|c|c|c|}
\hline \multirow[b]{2}{*}{ Subjects... } & \multicolumn{5}{|c|}{ GOS } & \multicolumn{5}{|c|}{ Placebo (MD) } & \multirow[b]{2}{*}{ SEM } \\
\hline & 1 & 5 & 6 & 8 & 12 & 2 & 3 & 7 & 9 & 11 & \\
\hline Total bacteria & $10 \cdot 4$ & $10 \cdot 1$ & $10 \cdot 2$ & $10 \cdot 5$ & 9.5 & $10 \cdot 4$ & $10 \cdot 4$ & $10 \cdot 4$ & $10 \cdot 4$ & $10 \cdot 4$ & 0.1 \\
\hline Bifidobacterium spp. & $9 \cdot 8$ & 9.4 & $10 \cdot 1$ & $9 \cdot 7$ & 9.5 & $10 \cdot 3$ & $9 \cdot 6$ & $7 \cdot 5$ & $10 \cdot 0$ & $9 \cdot 4$ & 0.3 \\
\hline
\end{tabular}

GOS, galacto-oligosaccharides; MD, maltodextrin.

* Samples collected at screening (1-7 d before day 0$)$. 

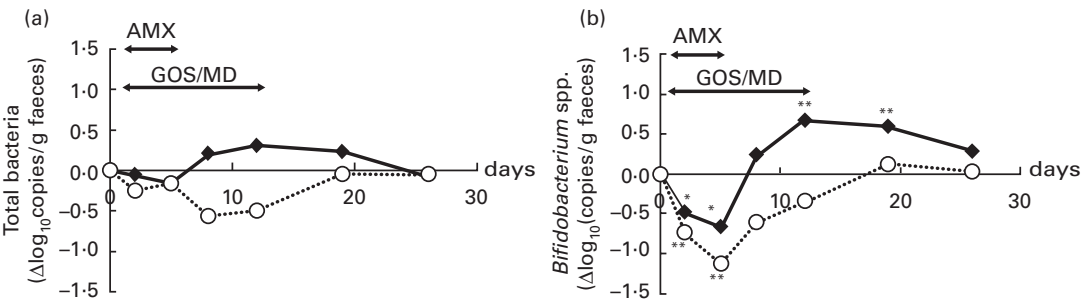

Fig. 2. Variation in the number of (a) total bacteria and (b) Bifidobacterium spp. in healthy adult subjects during and after treatment with amoxicillin (AMX) and galacto-oligosaccharides (GOS) ( $n 5$ until day $5 ; n 4$ after day $5 ;-)$ ) or AMX and maltodextrin (MD (placebo); $n 5 ;-0$ ). Values are means of the normalised log 10 copies/g faeces. Mean value was significantly different from that on day $0:{ }^{*} P<0.10$ (trend), ${ }^{\star \star} P<0.05$. For total bacteria: SEM $0.2, P_{\text {day }}=0.89, P_{\text {treatment }}=0.62$ and $P_{\text {interaction }}=0.41$. For Bifidobacterium spp.: SEM 0.3, $P_{\text {day }}=0.001, P_{\text {treatment }}=0.09$ and $P_{\text {interaction }}=0.10$.

the placebo group (Fig. 4(d)). On an individual level, the levels of some bacterial groups (mainly Bacteroides) also differed from the initial levels (see online supplementary Fig. S2).

\section{Effect of galacto-oligosaccharides on the metabolic activity of the microbiota}

The effect of GOS on the metabolic activity of AMX-disrupted microbiota was determined by measuring the levels of SCFA and intermediate OA, as well as the levels of monosaccharides and oligosaccharides (including disaccharides), in the faecal samples.

Levels of organic acids in the faecal samples. The levels of OA (SCFA and intermediate OA) were measured in the faecal samples of subjects treated with AMX and GOS or placebo (Fig. 6). During phase 0 (before the treatment), the total amount of $\mathrm{OA}$ in the faecal samples was similar in both groups, about 89 (SEM 13) $\mu \mathrm{mol} / \mathrm{g}$ faeces. The molar proportion (\%) of acetate:propionate:butyrate:valerate:succinate: lactate was about 59:20:16:4:0:0 in both groups.

Despite the small number of subjects, the mixed-model ANOVA revealed significant effects of day, treatment and day $\times$ treatment interaction on the variable 'butyrate' $(P=0.02)$ and per day on the variable 'succinate' $(P=0 \cdot 01)$. Subsequently, least significant difference post boc tests were carried out on these variables. During phase 1 (AMX + GOS/MD; days 2 and 5), the levels of succinate increased on day 2 in the GOS group (20 (SEM 3) $\mu \mathrm{mol} / \mathrm{g}$ faeces; $P<0.05$ ) and on day 5 in the placebo group (13 (sem 3) $\mu \mathrm{mol} / \mathrm{g}$ faeces; $P<0.05$ ) when compared with those on day 0 ( 0 (SEM 3$) \mu \mathrm{mol} / \mathrm{g}$ faeces). The proportion of succinate reached up to $22 \%$ of the total OA amount.
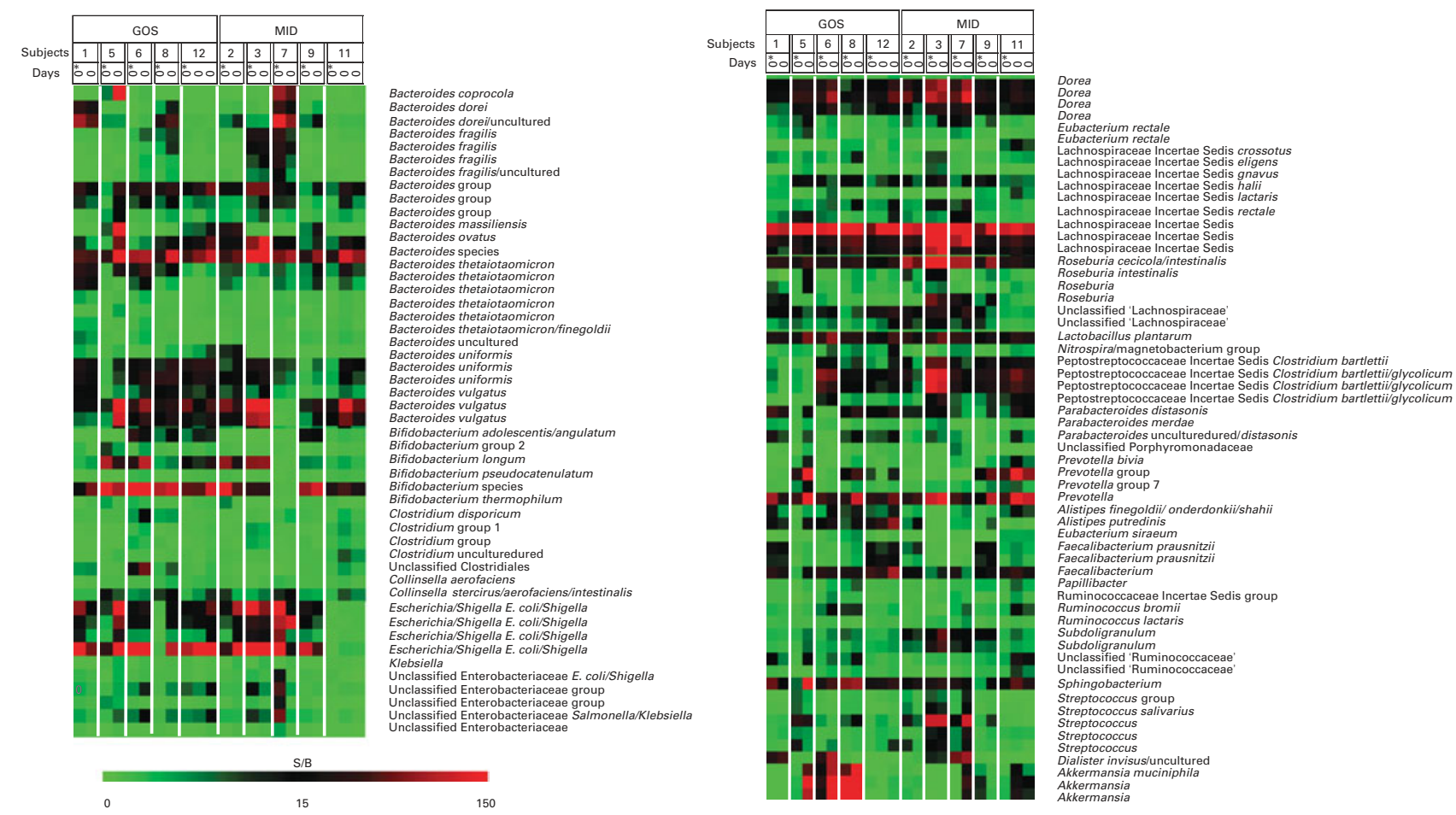

Fig. 3. Bacterial fingerprints of the microbiota of healthy adult subjects before the treatment (day $0^{*}$ and day 0 ) obtained with the I-Chip. Signal compared with the background (S/B): green - below the detectable level; black - medium abundance; red - high abundance. ${ }^{\star}$ Samples collected at screening (1-7 d before day 0 ). Analysis of day 0 sample was carried out in duplicate for subjects 11 and 12. GOS, galacto-oligosaccharides; MD, maltodextrin (placebo). 
(a)

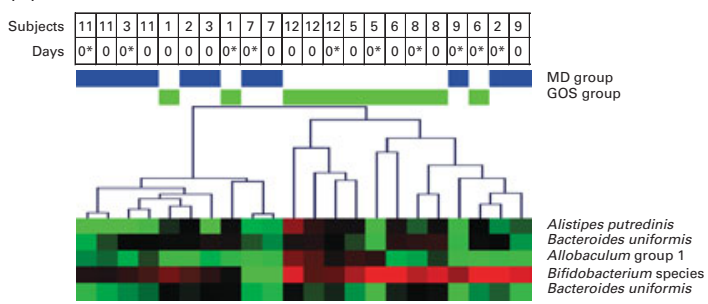

(c)

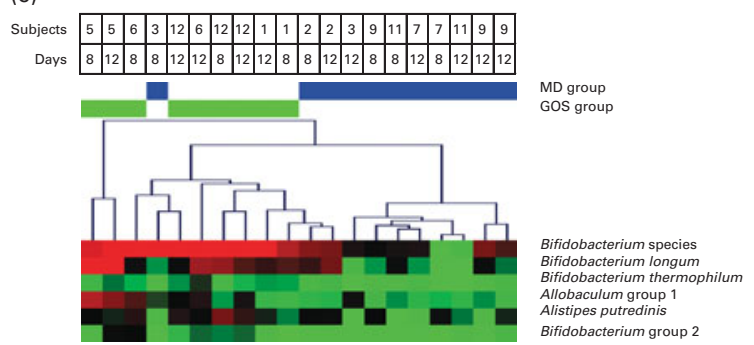

(b)

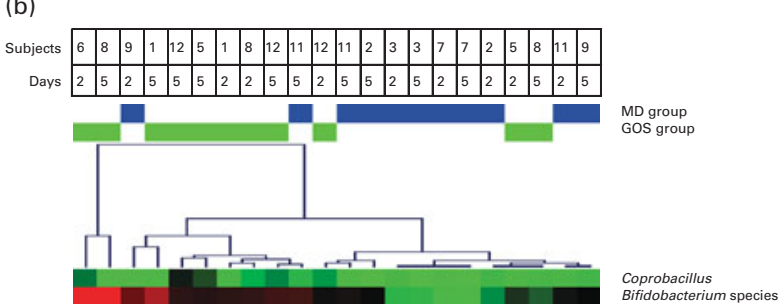

(d)

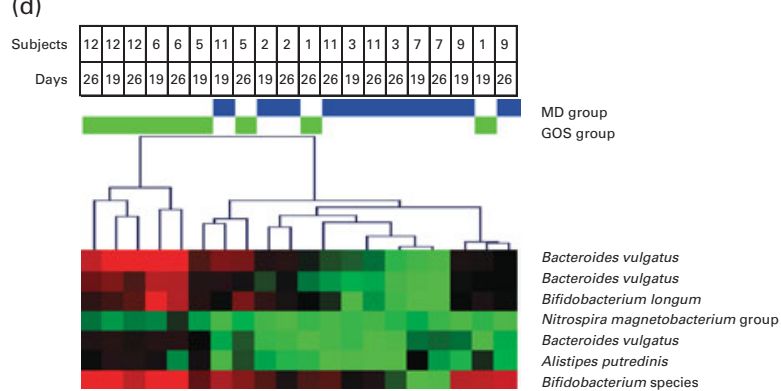

Fig. 4. Bacterial groups significantly different between the galacto-oligosaccharide (GOS) and placebo (maltodextrin; MD) groups at different periods of the treatment as detected by significance analysis of microarrays. A total of four periods were defined: (a) phase 0 (before the treatment); (b) phase 1 (amoxicillin (AMX) + GOS/MD); (c) phase 2 (GOS/MD); (d) phase 3 (follow-up period). Signal compared with the background (S/B): green - below the detectable level; black - medium abundance; red - high abundance. * Samples collected at screening (1-7d before day 0 ). Analyses of day 0 , day 5 , day 12 and day 26 samples were carried out in duplicate for subjects 11 and 12 .

The levels of succinate increased in both groups due to the action of AMX. During phase 2 (GOS/MD; days 8 and 12), the levels of butyrate on day 8 were higher in the GOS group than in the placebo group (26 (SEM 5) v. 9 (SEM 5) $\mu \mathrm{mol} / \mathrm{g}$ faeces; $P<0.05$ ), although not detected to be different on day 12 anymore. During phase 3 (follow-up period; days 19 and 26), the levels of SCFA and intermediate OA were similar to their respective initial levels in both the GOS and placebo groups. Surprisingly, the levels of butyrate were higher on day 19 (26 (sem 5) $\mu \mathrm{mol} / \mathrm{g}$ faeces; $P<0 \cdot 05)$ than on day 0 in the GOS group (11 (sEm 5) $\mu \mathrm{mol} / \mathrm{g}$ faeces).

Levels of monosaccharides and oligosaccharides in the faecal samples. The levels of monosaccharides and oligosaccharides (including disaccharides) were measured in the faecal samples using high-performance anion-exchange chromatography and expressed in $\mathrm{mg}$ saccharides (lactose equivalent) $/ \mathrm{g}$ faeces (Fig. 7). Before the treatment, the level of saccharides (85\% monosaccharides and 15\% oligosaccharides) in the faecal sample was 23 (SEM 4) $\mathrm{mg} / \mathrm{g}$ faeces in both the GOS and placebo groups. The mixed-model ANOVA did not reveal significant differences between the treatments and for the day $\times$ treatment interaction, indicating no differences between the levels of saccharides in the GOS groups and those in the placebo group. Nevertheless, the model revealed significant effects of time on the levels of monomers $(P=0 \cdot 04)$, oligosaccharides $(P<0 \cdot 001)$ and total carbohydrates
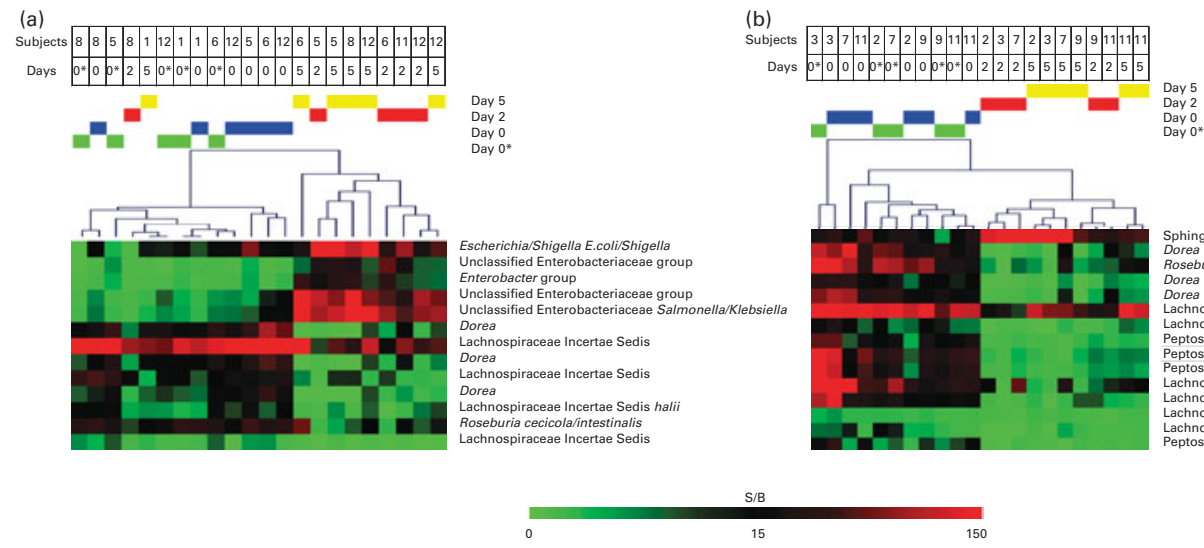

Fig. 5. Bacterial groups significantly different between phase 0 and phase 1 of the treatment in the (a) galacto-oligosaccharide (GOS) and (b) placebo (maltodextrin; MD) groups as detected by significance analysis of microarrays. Signal compared with the background (S/B): green - below the detectable level; black medium abundance; red - high abundance. * Samples collected at screening (1-7d before day 0$)$. Analyses of day 0 , day 5 , day 12 and day 26 samples were carried out in duplicate for subjects 11 and 12 . 


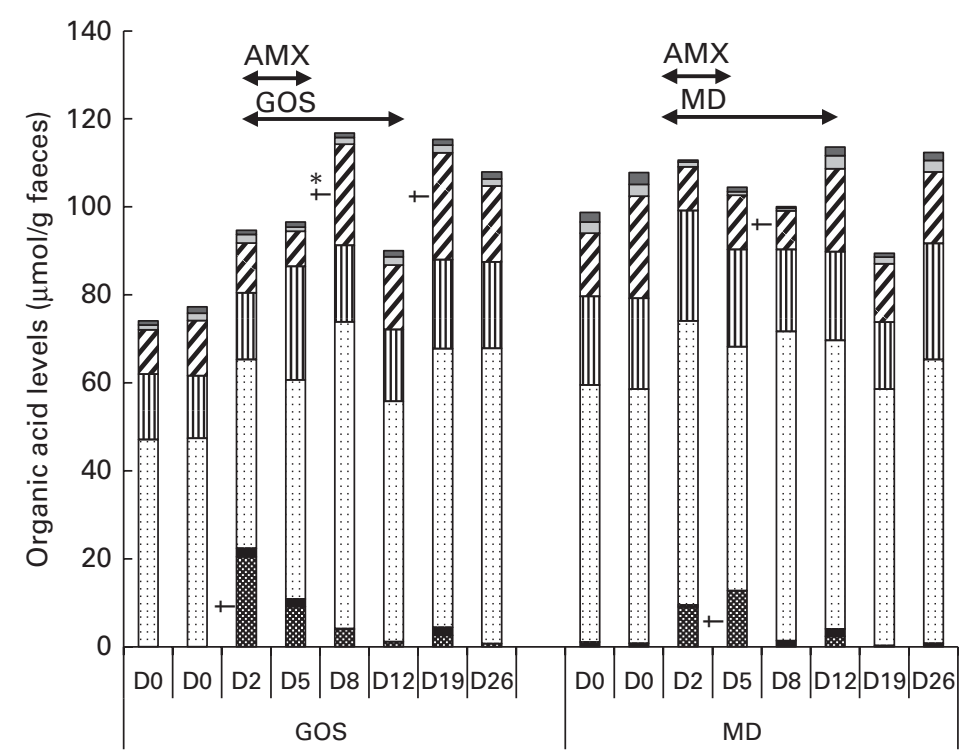

Fig. 6. Levels of organic acids in the faecal samples of healthy adult subjects receiving treatment with amoxicillin (AMX) and galacto-oligosaccharides (GOS) ( $n 5$ until day $5 ; n 4$ after day 5) or AMX and placebo (maltodextrin; MD; $n 5$ ). Values are means of $\mu \mathrm{mol} / \mathrm{g}$ faeces. * Mean value was significantly different from that obtained during the placebo treatment $(P<0.05)$. † Mean value was significantly different from that on day 0 within the treatment $(P<0.05)$. $\square$, Valerate

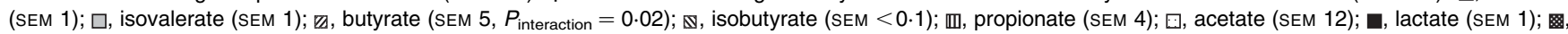
succinate (SEM $3, P_{\text {day }}=0 \cdot 01$ ). D, day.

$(P<0 \cdot 001)$. The levels of monosaccharides, oligosaccharides and total saccharides were higher on day 2 than on day 0 in the GOS group $(P<0 \cdot 05)$. The proportion of monosaccharide increased to $30 \%$ in the GOS group on day 2 .

\section{Discussion}

\section{Baseline conditions}

Before the treatment (phase 0; days $0^{*}$ and 0 ), subjects from both groups had a healthy complex microbiota composition. Individual diversity in the microbiota was observed at the species level, as reported previously ${ }^{(24)}$. The number of total bacteria $\left(10^{10.3}\right.$ copies/g faeces) was in the lower range of what is usually detected $\left(10^{9}-10^{12}\right.$ colony-forming units/ml $)^{(25,26)}$, while the number of Bifidobacterium spp. $\left(10^{9.5}\right.$ copies/g faeces) was in line with that reported in the literature $^{(27)}$. As a result, the estimated proportion of bifidobacteria (17\%) was rather high when compared with that reported in the literature $(\leq 10 \%)^{(28)}$. The discrepancy is most probably due to the low response of the primers used for total bacteria. Nevertheless, as the same primers were used for all the samples, the results obtained for the two intervention groups were similar. Regarding the activity of the microbiota before the treatment, the total amounts of OA (102 (sEM 15) $\mu \mathrm{mol} / \mathrm{g}$ faeces) and their relative proportions (acetate:butyrate:propionate:valerate 59:20:16:4) in the faecal samples were in line with those reported in the literature, about $100 \mu \mathrm{mol} / \mathrm{g}$ faeces of total SCFA ${ }^{(29)}$, a low amount of intermediate OA and a molar ratio of acetate:butyrate: propionate of $60: 20: 20^{(30)}$.
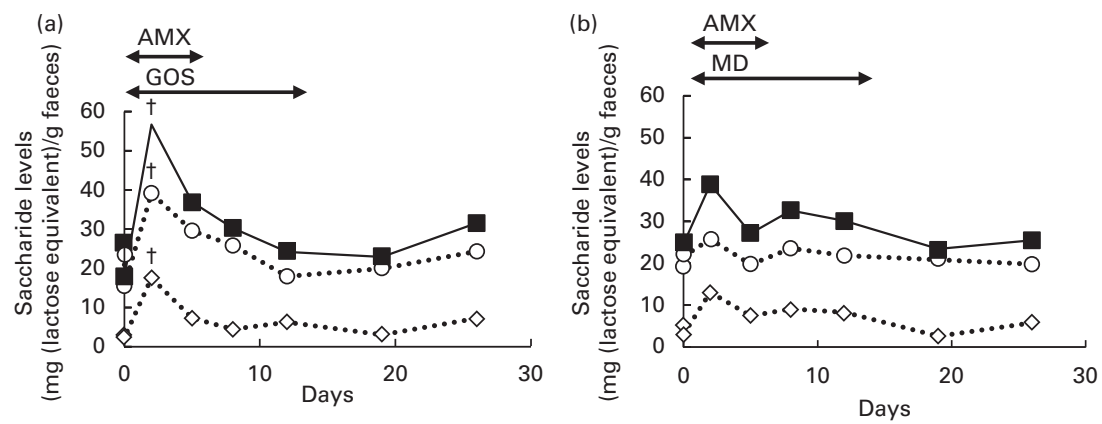

Fig. 7. Levels of monosaccharides and oligosaccharides (including disaccharides) in the faecal samples of healthy adult subjects receiving treatment with (a) amoxicillin (AMX) and galacto-oligosaccharides (GOS) or (b) AMX and placebo (maltodextrin; MD) as measured by high-performance anion-exchange chromatography. Values are means of $\mathrm{mg}$ saccharide (lactose equivalent) $/ \mathrm{g}$ faeces. † Mean value was significantly different from that on day 0 within the treatment $(P<0.05)$. O., Monomers (SEM 0.2, $\left.P_{\text {day }}=0.04, P_{\text {treatment }}=0.54, P_{\text {interaction }}=0.36\right) ; . \diamond \cdots$, oligomers $\left(\right.$ SEM $\left.0.3, P_{\text {day }}<0.001, P_{\text {treatment }}=0.93, P_{\text {interaction }}=0.73\right) ;$ - , total concentration (SEM $0.3, P_{\text {day }}<0.001, P_{\text {treatment }}=0.71, P_{\text {interaction }}=0.43$ ). 


\section{Effect of galacto-oligosaccharides on the microbiota during amoxicillin treatment}

Microbiota composition. Although a low dose of AXM and a short duration of AMX treatment were used, changes in the composition of the microbiota were expected ${ }^{(31)}$. In both the placebo and GOS groups, the mean number of total bacteria remained stable during AMX treatment, which is in contradiction to the results of previous in vivo studies ${ }^{(32,33)}$. On an individual level, a decrease of up to $2 \log _{10}$ was observed in four subjects (two subjects from each group) on different days (day 2, 5 or 8 after the discontinuation of the treatment) (data not shown). This indicates that the response to an antibiotic treatment depends on the individual ${ }^{(34)}$. Differences among individuals might be due to a variation in the level of amoxicillin absorbed into the blood, for instance, due to their diet ${ }^{(35)}$. Nevertheless, changes in the composition of the microbiota were observed in each subject between phase 1 and phase 0 (Fig. 5 and Fig. S2). Therefore, part of the AMX dose ingested certainly reached the colon and affected part of the bacterial population. The number of total bacteria did not change probably because the nonaffected bacteria grew and occupied free sites in the colon, resulting in a shift in the composition rather than a decrease in the abundance of the population ${ }^{(36)}$.

A shift in the composition was observed for Lachnospiraceae, Enterobacteriaceae and Bifidobacterium spp. The decrease in the relative abundance of Lachnospiraceae and Bifidobacterium spp. and the increase in the relative abundance of Enterobacteriaceae observed in three of the five subjects in the placebo group due to AMX treatment are consistent with the literature ${ }^{(31)}$. Although no significant differences were observed between the placebo and GOS groups, the intake of GOS by subjects receiving AMX treatment appeared to limit the decrease in the abundance of Bifidobacterium spp. and not to prevent either the decrease in the abundance of Lachnospiraceae or the growth of Enterobacteriaceae. The low levels of Lachnospiraceae as well as the higher levels of Bifidobacterium spp. in the GOS group compared with the levels in the placebo group are in line with the results of a previous in vitro study ${ }^{(7)}$. The level of bifidobacteria measured in vitro at $24 \mathrm{~h}$ of fermentation was already similar to or higher than that measured at $t=0$, whereas it was still lower than the initial levels during AMX treatment in the in vivo study. The difference in the rate of recovery might be explained by the use of a diluted system in vitro. It is also possible that the level of bifidobacteria measured during in vitro fermentation was higher because of the attenuation of the effect of AMX over time in an in vitro assay. The relatively high abundance of Enterobacteriaceae is in contradiction to the data obtained in a previous in vitro study ${ }^{(7)}$. This discrepancy might be explained by a difference in the initial level of Enterobacteriaceae in vitro (low) when compared with that in vivo (high). Possibly, the difference between the initial levels resides in the variability in the composition of the microbiota between the inocula. It might also be that the pre-culture step of the in vitro study preferably induced the growth of Enterobacteriaceae ${ }^{(37)}$. Furthermore, the discrepancy might be due to a different contact mechanism between the antibiotic and the bacteria in the batch system (stronger) when compared with that in the colon (less severe because of the presence of many villi). Eventually, the relatively high abundance of Enterobacteriaceae should be confirmed by quantification, as microarrays only provide data on relative changes in the composition of the microbiota.

Overall, it can be concluded that GOS intake did not significantly prevent the occurrence of changes in the composition of the microbiota observed during AMX treatment, although GOS appeared to limit the decrease in the abundance of bifidobacteria.

Microbiota metabolic activity. The disruption of the composition of the microbiota upon AMX treatment was also reflected by the changes observed in the metabolic activity. The levels of succinate increased on day 5 in the placebo group when compared with those on day 0 (13 (SEM 3) v. 0 ( $\mathrm{sEm} 3$ ) $\mu \mathrm{mol} / \mathrm{g}$ faeces; $P<0.05$ ). In addition, the levels of monosaccharides and oligosaccharides appeared to increase on day 2 in the placebo group when compared with those on day 0 , although not significantly. Most probably, the metabolic activity of the bacteria, including enzyme synthesis and fermentation activity, was reduced upon antibiotic treatment, resulting in a lower degradation of the oligosaccharides available ${ }^{(38,39)}$, an accumulation of monosaccharides, and a lower conversion of the intermediate OA into SCFA ${ }^{(9)}$. The changes in metabolic activity were also observed upon GOS intake, as reported previously in an in vitro study ${ }^{(7)}$. Higher levels of monosaccharides and oligosaccharides were measured on day 2 than on day 0 (39 (SEM 2) and 18 (SEM 2) v. 20 (SEM 2) and 3 (SEM 2) $\mathrm{mg} / \mathrm{g}$ faeces; $P<0 \cdot 05$ ), reflecting the fibre-enriched diet of the GOS group. In addition, the fermentation of GOS by bifidobacteria was reflected by the increase in lactate levels in the GOS group when compared with those in the placebo group (2 (SEM 1) $v$. 0 (SEM 1) $\mu \mathrm{mol} / \mathrm{g}$ faeces), although this increase was not found to be significant. As the cross-feeding network is disrupted due to the action of AMX, lactate is accumulated instead of being converted to SCFA, e.g. butyrate ${ }^{(40)}$. In conclusion, despite evidence for the partial utilisation of GOS by the microbiota, the overall metabolic activity of the microbiota was still significantly disturbed during AMX treatment.

\section{Effect of galacto-oligosaccharides on the microbiota after amoxicillin treatment}

After AMX treatment, the levels of bifidobacteria were higher in the GOS group than in the placebo group (+1 (SEM 0.2) $\log _{10}$ on day 12). Bifidobacteria species produce lactate and acetate $^{(40)}$. The higher levels of butyrate in the GOS group compared with the levels in the placebo group (26 (SEM 5) v. 9 (SEM 5) $\mu \mathrm{mol} / \mathrm{g}$ faeces; $P<0.05$ ) indicate that GOS intake also stimulated the recovery of other bacteria in the ecosystem, probably through cross-feeding on lactate and acetate ${ }^{(40)}$. The levels of total bacteria were higher in the GOS group than in the placebo group, although the difference was not found to be significant. This result might reflect the recovery of bifidobacteria and other groups of the microbial population, 
e.g. butyrate-producing bacteria in the GOS group. The growth of butyrate-producing bacteria, such as Eubacterium and Roseburia $^{(41)}$, was detected in the present study after the discontinuation of AMX treatment (see online supplementary Fig. S1). Overall, the recovery of the composition and metabolic activity of the microbiota occurred faster upon GOS intake than upon placebo intake after the discontinuation of AMX treatment. An increase in the levels of bifidobacteria in infants given a mixture of fructo-oligosaccharides and inulin has also been observed after the discontinuation of AMX treatment ${ }^{(33)}$. It, therefore, appears that the effects of GOS on the AMXdisrupted microbiota are beneficial after the discontinuation of AMX treatment rather than during the treatment.

\section{Resilience of the microbiota}

In the follow-up period (3 weeks after the discontinuation of AMX treatment), the levels of bifidobacteria as well the SCFA profile and the amounts of monosaccharides and oligosaccharides in faeces returned to the initial levels. The recovery of the composition of the microbiota has been reported to occur within a month in a previous study ${ }^{(10)}$. In the present study, we showed that the recovery of the metabolic activity of the microbiota occurs shortly after the discontinuation of AMX treatment, despite the composition being still altered at that time point. This finding illustrates the reported redundancy of the functionality of the microbiota ${ }^{(28)}$.

Despite a high percentage of resilience being observed in the bacterial fingerprints, individual differences at the species level were detected, especially for the Bacteroides group. These differences at the species level might indicate a longterm impact of antibiotics on the microbiota ${ }^{(42)}$. Long-term impacts have been reported to increase with increasing exposure events and short resilience time between the subsequent treatments ${ }^{(34)}$.

\section{Effect of galacto-oligosaccharides on stool parameters and} gastrointestinal discomforts

In general, no effects of GOS on gastrointestinal discomforts, such as bloating, flatulence, heartburn and nausea, were reported by both the placebo and GOS groups. This was expected as the dose of GOS $(7.5 \mathrm{~g} / \mathrm{d})$ was chosen in the range where GOS has been reported to have a bifidogenic effect but limited side effects (from 2.5 to $10 \cdot 0 \mathrm{~g} / \mathrm{d}$ ) ${ }^{(15)}$.

On an individual level, the two subjects who reported one loose bowel movement or diarrhoea once belonged to the GOS group. Several factors could have caused this effect, among which is the disruption of the composition of the microbiota by AMX treatment (observed decrease in the number of Bifidobacterium spp. and increase in the number of Enterobacteriaceae). It can also be speculated that the amount of GOS added to the unknown amount of fibre already present in the subjects' diet (non-controlled diet) might have been too high to be handled by the AMX-disturbed microbiota, resulting in an osmotic diarrhoea. This would be in line with the high levels of saccharides observed on day 2 in the GOS group $(57 \mathrm{mg} / \mathrm{g}$ faeces; Fig. 7(a)). Furthermore, consumption of a particular or a spoilt product or another change in the diet of the subjects could be the reason.

\section{Concluding remarks}

In the present in vivo study, the potential of GOS to limit the decrease in the abundance of bifidobacteria during AMX treatment and to stimulate the growth of bifidobacteria after the discontinuation of AMX treatment was shown. Considering SCFA profiles and monosaccharide and oligosaccharide levels in faeces, it was also shown that GOS intake stimulates the recovery of the metabolic activity of the microbiota after the discontinuation of AMX treatment rather than during the treatment. The present study provides data that confirm the results obtained in a previous in vitro study ${ }^{(7)}$ and, thereby, justify further research involving targeted populations, such as patients, infants and elderly rather than healthy adults.

\section{Supplementary material}

To view supplementary material for this article, please visit http://dx.doi.org/10.1017/S0007114514001135

\section{Acknowledgements}

The authors thank the subjects for participating in the study.

The present study was jointly funded by the European Union, the European Regional Development Fund and The Ministry of Economic Affairs, Agriculture and Innovation, Peaks in the Delta, the Municipality of Groningen, and the Province of Groningen as well as the Dutch Carbohydrate Competence Center (CCC-WP2) and by FrieslandCampina.

The authors' contributions are as follows: S. E. L., M. H. C. S., M. M., A. N. and H. A. S. designed the study; S. E. L. conducted the study and performed the chemistry-related analyses; H. R. performed the microbiology-related analyses; S. E. L. analysed the data and wrote the article along with M. H. C. S., M. M., F. H. J. S., H. G., A. N. and H. A. S. All the authors read and approved the final version of the manuscript.

None of the authors has any conflicts of interest to declare.

\section{References}

1. European Centre for Disease Prevention and Control (2010) Surveillance of antimicrobial consumption in Europe. http:// ecdc.europa.eu/en/publications/Publications/antimicrobialantibiotic-consumption-ESAC-report-2010-data.pdf (cited September 2013).

2. McFarland LV (2008) Antibiotic-associated diarrhea: epidemiology, trends and treatment. Future Microbiol 3, 563-578.

3. Gibson GR, Probert HM, Van Loo J, et al. (2004) Dietary modulation of the human colonic microbiota: updating the concept of prebiotics. Nutr Res Rev 17, 259-275.

4. Macfarlane S, Macfarlane GT \& Cummings JH (2006) Review Article: prebiotics in the gastrointestinal tract. Aliment Pharmacol Ther 24, 701-714.

5. Saulnier DM, Kolida S \& Gibson GR (2009) Microbiology of the human intestinal tract and approaches for its dietary modulation. Curr Pharm Des 15, 1403-1414. 
6. Macfarlane GT \& Macfarlane LE (2009) Acquisition, evolution and maintenance of the normal gut microbiota. Dig Dis 27, 90-98.

7. Ladirat SE, Schuren FH, Schoterman MH, et al. (2014) Impact of galacto-oligosaccharides on the gut microbiota composition and metabolic activity upon antibiotic treatment during in vitro fermentation. FEMS Microbiol Ecol 87, 41-51.

8. Alonso VR \& Guarner F (2013) Linking the gut microbiota to human health. Br J Nutr 109, S21-S26.

9. Willing BP, Russell SL \& Finlay BB (2011) Shifting the balance: antibiotic effects on host-microbiota mutualism. Nat Rev Microbiol 9, 233-243.

10. De La Cochetière MF, Durand T, Lepage P, et al. (2005) Resilience of the dominant human fecal microbiota upon shortcourse antibiotic challenge. J Clin Microbiol 43, 5588-5592.

11. Jeong SH, Song YK \& Cho JH (2009) Risk assessment of ciprofloxacin, flavomycin, olaquindox and colistin sulfate based on microbiological impact on human gut biota. Regul Toxicol Pharmacol 53, 209-216.

12. Jalanka-Tuovinen J, Salonen A, Nikkilä J, et al. (2011) Intestinal microbiota in healthy adults: temporal analysis reveals individual and common core and relation to intestinal symptoms. PLOS ONE 6, e23035.

13. Gibson GR, Beatty ER, Wang X, et al. (1995) Selective stimulation of bifidobacteria in the human colon by oligofructose and inulin. Gastroenterology 108, 975-982.

14. SWAB/RIVM (2009) NethMap report. http://www.swab.nl/ swab/cms3.nsf/uploads/1D61A8F6E60555F3C125763900414 B7B/\$FILE/nethmap2009_21-9-2009.pdf (cited September 2013).

15. Davis LMG, Martínez I, Walter J, et al. (2010) A dose dependent impact of prebiotic galactooligosaccharides on the intestinal microbiota of healthy adults. Int $J$ Food Microbiol 144, 285-292.

16. Ladirat SE, Schols HA, Nauta A, et al. (2013) High-throughput analysis of the impact of antibiotics on the human intestinal microbiota composition. J Microbiol Methods 92, 387-397.

17. François IEJA, Lescroart O, Veraverbeke WS, et al. (2012) Effects of a wheat bran extract containing arabinoxylan oligosaccharides on gastrointestinal health parameters in healthy adult human volunteers: a double-blind, randomised, placebo-controlled, cross-over trial. Br J Nutr 108, 2229-2242.

18. Wanders AJ, Mars M, Borgonjen-van den Berg $\mathrm{KJ}$, et al. (2013) Satiety and energy intake after single and repeated exposure to gel-forming dietary fiber: post-ingestive effects Int $J$ Obes (Lond) (Epublication ahead of print version 13 September 2013).

19. World Health Organization (2013) Diarrhoea. http://www who.int/topics/diarrhoea/en/ (cited September 2013).

20. Crielaard W, Zaura E, Schuller AA, et al. (2011) Exploring the oral microbiota of children at various developmental stages of their dentition in the relation to their oral health. $B M C$ Med Genomics 4, 22

21. Albrecht S, Schols HA, Van Zoeren D, et al. (2011) Oligosaccharides in feces of breast- and formula-fed babies Carbohydr Res 346, 2173-2181.

22. Jonathan MC, Bosch G, Schols HA, et al. (2013) Separation and identification of individual alginate oligosaccharides in the feces of alginate-fed pigs. J Agric Food Chem 61, $553-560$

23. Saeed AI, Sharov V, White J, et al. (2003) TM4: a free, open-source system for microarray data management and analysis. Biotechniques 34, 374-378.

24. Eckburg PB, Bik EM, Bernstein CN, et al. (2005) Microbiology: diversity of the human intestinal microbial flora. Science 308, 1635-1638.
25. Filteau M, Matamoros S, Savard P, et al. (2013) Molecular monitoring of fecal microbiota in healthy adults following probiotic yogurt intake. PharmaNutrition 1, 123-129.

26. Blaut M \& Clavel T (2007) Metabolic diversity of the intestinal microbiota: implications for health and disease. $J$ Nutr 137, 751S-755S

27. Matsuki T, Watanabe K, Fujimoto J, et al. (2004) Quantitative PCR with 16S rRNA-gene-targeted species-specific primers for analysis of human intestinal bifidobacteria. Appl Environ Microbiol 70, 167-173

28. Turroni F, Ribbera A, Foroni E, et al. (2008) Human gut microbiota and bifidobacteria: from composition to functionality. Antonie van Leeuwenhoek 94, 35-50.

29. Macfarlane S \& Macfarlane GT (2003) Session: short-chain fatty acids. Regulation of short-chain fatty acid production. Proc Nutr Soc 62, 67-72.

30. Wong JMW, De Souza R, Kendall CWC, et al. (2006) Colonic health: fermentation and short chain fatty acids. J Clin Gastroenterol 40, 235-243.

31. Sullivan A (2001) Effect of antimicrobial agents on the ecological balance of human microflora. Lancet Infect Dis 1, 101-114.

32. Bartosch S, Fite A, Macfarlane GT, et al. (2004) Characterization of bacterial communities in feces from healthy elderly volunteers and hospitalized elderly patients by using realtime PCR and effects of antibiotic treatment on the fecal microbiota. Appl Environ Microbiol 70, 3575-3581.

33. Brunser O, Gotteland M, Cruchet $S$, et al. (2006) Effect of a milk formula with prebiotics on the intestinal microbiota of infants after an antibiotic treatment. Pediatr Res 59, 451-456.

34. Dethlefsen L \& Relman DA (2011) Incomplete recovery and individualized responses of the human distal gut microbiota to repeated antibiotic perturbation. Proc Natl Acad Sci US A 108, 4554-4561.

35. Welling P (1977) Influence of food and diet on gastrointestinal drug absorption: a review. J Pharmacokinet Biopharm $\mathbf{5}$, 291-334.

36. Sekirov I, Tam NM, Jogova M, et al. (2008) Antibioticinduced perturbations of the intestinal microbiota alter host susceptibility to enteric infection. Infect Immun $\mathbf{7 6}$, $4726-4736$.

37. Kovatcheva PP, Zoetendal EG, Venema K, et al. (2009) Review: tools for the tract: understanding the functionality of the gastrointestinal tract. Therap Adv Gastroenterol 2, s9-s22.

38. Newton DF, MacFarlane S \& MacFarlane GT (2013) Effects of antibiotics on bacterial species composition and metabolic activities in chemostats containing defined populations of human gut microorganisms. Antimicrob Agents Chemother 57, 2016-2025.

39. Yap IKS, Li JV, Saric J, et al. (2008) Metabonomic and microbiological analysis of the dynamic effect of vancomycininduced gut microbiota modification in the mouse. I Proteome Res 7, 3718-3728.

40. Belenguer A, Duncan SH, Calder AG, et al. (2006) Two routes of metabolic cross-feeding between Bifidobacterium adolescentis and butyrate-producing anaerobes from the human gut. Appl Environ Microbiol 72, 3593-3599.

41. Louis P \& Flint HJ (2009) Diversity, metabolism and microbial ecology of butyrate-producing bacteria from the human large intestine. FEMS Microbiol Lett 294, 1-8.

42. Jernberg C, Löfmark S, Edlund C, et al. (2007) Long-term ecological impacts of antibiotic administration on the human intestinal microbiota. ISME J 1, 56-66. 